\title{
A deficit scaling algorithm for the minimum flow problem
}

\author{
LAURA CIUPALA \\ Department of Computer Science, Faculty of Mathematics \& Informatics, \\ Transilvania University of Braşov, Romania \\ e-mail: laura_ciupala@yahoo.com
}

MS received 30 April 2005; revised 18 January 2006

\begin{abstract}
In this paper, we develop a new preflow algorithm for the minimum flow problem, called deficit scaling algorithm. This is a special implementation of the generic preflow algorithm for the minimum flow problem developed by Ciurea and Ciupală earlier. The bottleneck operation in the generic preflow algorithm is the number of noncancelling pulls. Using the scaling technique (i.e. selecting the active nodes with sufficiently large deficits), we reduce the number of noncancelling pulls to $O\left(n^{2} \log \bar{c}\right)$ and obtain an $O\left(n m+n^{2} \log \bar{c}\right)$ algorithm.
\end{abstract}

Keywords. Network flow; network algorithms; minimum flow problem; scaling technique.

\section{Introduction}

The literature on network flow problem is extensive. Over the past 50 years researchers have made continuous improvements to algorithms for solving several classes of problems. From the late 1940s through the 1950s, researchers designed many of the fundamental algorithms for network flow, including methods for maximum flow and minimum cost flow problems. In the next few decades, there were many research contributions towards improving the computational complexity of network flow algorithms by using enhanced data structures, techniques of scaling the problem data etc.

Although it has its own applications, the minimum flow problem was not treated as often as the maximum flow and the minimum cost flow problem. There are many problems that occur in economy that can be reduced to minimum flow problems. We present here the machine setup problem, which can be elegantly solved by determining a minimum flow in a network. A job shop needs to perform $p$ tasks on a particular day. The start time $\pi(i)$ and the end time $\pi^{\prime}(i)$ for each task $i, i=1, \ldots, p$ is known. The workers must perform these tasks according to this schedule so that exactly one worker performs each task. A worker cannot work on two jobs at the same time. We know the setup time $\pi_{0}(i, j)$ required for a worker to go from task $i$ to task $j$. We wish to find the minimum number of workers to perform the tasks. We can formulate this problem as a minimum flow problem in a network (for details see Ciurea \& Ciupală 2004) and the value of the minimum flow in this network is then the minimum number of workers. 
The minimum flow problem in a network can be solved in two phases:

(1) establishing a feasible flow, if there is one,

(2) from a given feasible flow, establish the minimum flow.

The problem of determining a feasible flow can be reduced to a maximum flow problem (for details see Ahuja et al 1993).

For the second phase of the minimum flow problem there are three approaches:

(1) using decreasing path algorithms (see Ciurea \& Ciupală 2001, 2004),

(2) using preflow algorithms (see Ciurea \& Ciupală 2001, 2004, Ciupală \& Ciurea 2003)

(3) finding a maximum flow from the sink node to the source node in the residual network (see Bang-Jensen \& Gutin 2001, Ciupală \& Ciurea 2001).

The preflow algorithms for the minimum flow are more efficient than the decreasing path algorithms. Ciurea \& Ciupală (2004) presented a generic preflow algorithm that runs in $O\left(n^{2} m\right)$ time and two special implementations of it: a FIFO preflow algorithm that runs in $O\left(n^{3}\right)$ time and a highest preflow algorithm that runs in $O\left(n^{2} \sqrt{m}\right)$ time.

In this paper, using the scaling technique, we develop a new algorithm for the minimum flow problem, called deficit scaling algorithm. This algorithm is a special implementation of the generic preflow algorithm for minimum flow (described by Ciurea \& Ciupală 2001, 2004) that selects always an active node with sufficiently large deficit and runs in $O\left(n m+n^{2} \log \bar{c}\right)$ time.

\section{Notation and definition}

We consider a capacitated network $G=(N, A, l, c, s, t)$ with a nonnegative capacity $c(i, j)$ and a nonnegative lower bound $l(i, j)$ associated with each $\operatorname{arc}(i, j) \in A$. We distinguish two special nodes in the network $G$ : a source node $s$ and a sink node $t$.

Let $n=|N|, m=|A|$ and $\bar{c}=\max \{c(i, j) \mid c(i, j) \in A\}$.

A flow is a function $f: A \rightarrow R_{+}$satisfying the next conditions:

$$
\begin{aligned}
& f(i, N)-f(N, i)= \begin{cases}v, & i=s, \\
0, & i \neq s, t, \\
-v, & i=t,\end{cases} \\
& l(i, j) \leq f(i, j) \leq c(i, j), \quad \forall(i, j) \in A,
\end{aligned}
$$

for some $v \geq 0$, where

$$
f(i, N)=\sum_{j \mid(i, j) \in A} f(i, j)
$$

and

$$
f(N, i)=\sum_{j \mid(j, i) \in A} f(j, i) .
$$

We refer to $v$ as the value of the flow $f$. 
The minimum flow problem is to determine a flow $f$ for which $v$ is minimized.

For the minimum flow problem, a preflow is a function $f: A \rightarrow R_{+}$satisfying the further conditions:

$$
\begin{aligned}
& f(i, N)-f(N, i) \leq 0, \quad \forall i \in N \backslash\{s, t\}, \\
& l(i, j) \leq f(i, j) \leq c(i, j) \forall(i, j) \in A .
\end{aligned}
$$

Let $f$ be a preflow. We define the deficit of any node $i \in N \backslash\{s, t\}$ in the following manner:

$$
e(i)=f(i, N)-f(N, i) .
$$

Thus, for the minimum flow problem, for any preflow $f$, we have $e(i) \leq 0, \forall i \in N \backslash\{s, t\}$.

We say that a node $i \in N \backslash\{s, t\}$ is active if $e(i)<0$ and balanced if $e(i)=0$. By convention, the source node $s$ and the sink node $t$ are never active.

A preflow $f$ for which

$$
e(i)=0, \forall i \in N \backslash\{s, t\},
$$

is a flow. Consequently, a flow is a particular case of preflow.

For the minimum flow problem, the residual capacity $r(i, j)$ of any arc $(i, j) \in A$, with respect to a given preflow $f$, is given by $r(i, j)=c(j, i)-f(j, i)+f(i, j)-l(i, j)$. By convention, if $(j, i) \notin A$ then we add $\operatorname{arc}(j, i)$ to the set of $\operatorname{arcs} A$ and we set $l(j, i)=0$ and $c(j, i)=0$. The residual capacity of the arc $(i, j)$ represents the maximum amount of flow from the node $i$ to node $j$ that can be cancelled. The network $G_{f}=\left(N, A_{f}\right)$ consisting only of the arcs with positive residual capacity is referred to as the residual network (with respect to the preflow $f$ ).

In the residual network $G_{f}=\left(N, A_{f}\right)$, the distance function $d: N \rightarrow \mathbf{N}$ with respect to a given preflow $f$ is a function from the set of nodes to the nonnegative integers. We say that a distance function is valid if it satisfies the following conditions:

$$
\begin{aligned}
& d(s)=0 \\
& d(j) \leq d(i)+1, \forall(i, j) \in A_{f} .
\end{aligned}
$$

We refer to $d(i)$ as the distance label of node $i$.

Lemma 1. (Ciurea \& Ciupală 2004) (a) If the distance labels are valid, the distance label $d(i)$ is a lower bound on the length of the shortest directed path from node s to node $i$ in the residual network.

(b) If $d(t) \geq n$, the residual network contains no directed path from the source node to the sink node.

We say that the distance labels are exact if for each node $i, d(i)$ equals the length of the shortest path from node $s$ to node $i$ in the residual network.

We refer to an arc $(i, j)$ from the residual network as an admissible arc if $d(j)=d(i)+1$; otherwise it is inadmissible.

\section{Deficit scaling algorithm}

Our algorithm is a special implementation of the generic preflow algorithm for minimum flow and, like all preflow algorithms for minimum flow, it maintains a preflow at every step and 
proceeds by pulling the deficits of the active nodes closer to the source node. For measuring closeness we use the exact distance labels. Consequently, pulling the deficits from the active nodes closer to the source node means decreasing flow on admissible arcs.

We refer to a pull of flow from node $j$ to node $i$ as a canceling pull if it deletes the arc $(i, j)$ from the residual network; otherwise it is a noncanceling pull.

Let $e_{\max }=\max \{-e(i) \mid i$ is an active node $\}$. The deficit dominator is the smaller integer $\bar{r}$ that is a power of 2 and satisfies $e_{\max } \leq \bar{r}$. We refer to a node $i$ with $e(i) \leq-r / 2$ as a node with large deficit and as a node with small deficit otherwise.

Our scaling deficit algorithm for the minimum flow always pulls flow from active nodes with sufficiently large deficits to nodes with sufficiently small deficits in order to not allow that a deficit becomes too large.

Thus, using the scaling technique (i.e. selecting active nodes with sufficiently large deficits) and imposing the rule that we pull flow to nodes with sufficiently small deficits, we obtain the following simple and efficient algorithm for the minimum flow problem:

\section{DEFICIT SCALING ALGORITHM;}

\section{BEGIN}

let $f$ be a feasible flow in network $G$;

compute the exact distance labels $d(\cdot)$ in the residual network $G_{f}$;

IF $t$ is not labeled THEN $\mathrm{f}$ is a minimum flow

ELSE

\section{BEGIN}

for each $\operatorname{arc}(i, t) \in A \mathrm{DO} f(i, t):=l(i, t)$;

$$
\begin{aligned}
& d(t):=n ; \\
& \bar{r}:=2^{\lceil\log \bar{c}\rceil} ;
\end{aligned}
$$

WHILE $\bar{r} \geq 1$ DO

\section{BEGIN}

WHILE the network contains an active node with a large deficit DO

\section{BEGIN}

among all nodes with large deficits, select a node $j$ with the smallest distance label;

IF the network contains an admissible arc $(i, j)$ THEN

pull $g=\min \{-e(j), r(i, j), \bar{r}+e(i)\}$ units of flow from node $j$ to node $i$;

$\operatorname{ELSE} d(j)=\min \left\{d(i) \mid(i, j) \in A_{f}\right\}+1$;

END;

$\bar{r}:=\bar{r} / 2$,

END;

END;

END. 
A pull of $g$ units of flow from node $j$ to node $i$ consists in the following operations:

$$
\begin{aligned}
& e(i):=e(i)-g, \\
& r(i, j):=r(i, j)-g, \\
& e(j):=e(j)+g \text { and } \\
& r(j, i):=r(j, i)+g .
\end{aligned}
$$

Let us refer to a phase of the algorithm during which $\bar{r}$ remains constant as a scaling phase and a scaling phase with a specific value of $\bar{r}$ as a $\bar{r}$-scaling phase.

Theorem 1. If there is a feasible flow in the network $G$, then the deficit scaling algorithm determines a minimum flow in $G$.

Proof. The algorithm starts with $\bar{r}:=2^{\lceil\log \bar{c}\rceil}, \bar{c} \leq \bar{r} \leq 2 \bar{c}$. During the $\bar{r}$-scaling phase, $e_{\max }$ might increase or decrease but it must meet the condition $\bar{r} / 2<e_{\max } \leq \bar{r}$. When $e_{\max } \leq \bar{r} / 2$ the algorithm halves the value of $\bar{r}$ and begins a new scaling phase. After $1+\lfloor\log \bar{c}\rfloor$ scaling phases, $e_{\max }$ becomes 0 and we obtain a minimum flow.

Actually, the algorithm terminates with optimal residual capacities. From these residual capacities we can determine a minimum flow in several ways. For example, we can make a variable change: For all arcs $(i, j)$, let $c^{\prime}(i, j)=c(i, j)-l(i, j), r^{\prime}(i, j)=r(i, j)$ and $f^{\prime}(i, j)=f(i, j)-l(i, j)$. The residual capacity of $\operatorname{arc}(i, j)$ is

$$
r(i, j)=c(j, i)-f(j, i)+f(i, j)-l(i, j) .
$$

Equivalently,

$$
r^{\prime}(i, j)=c^{\prime}(j, i)-f^{\prime}(j, i)+f^{\prime}(i, j) .
$$

We can compute the value of $f^{\prime}$ in the following way:

$$
f^{\prime}(i, j)=\max \left(r^{\prime}(i, j)-c^{\prime}(j, i), 0\right) .
$$

Converting back into the original variables, we obtain the following expressions:

$$
f(i, j)=l(i, j)+\max (r(i, j)-c(j, i)+l(j, i), 0), \text { for all arcs }(i, j) .
$$

Theorem 2. During each $\bar{r}$-scaling phase, the algorithm satisfies the following two conditions:

(1) each noncanceling pull decreases the flow by at least $\bar{r} / 2$ units

(2) $e_{\max } \leq \bar{r}$

Proof. (1) We consider a noncanceling pull on arc $(i, j)$. Since $(i, j)$ is an admissible arc, $d(j)=d(i)+1>d(i)$. But, $j$ is a node with a smallest distance label among all nodes with a large deficit. Thus, $e(j) \leq-\bar{r} / 2$ and $e(i)>-\bar{r} / 2$. Since this pull is a noncanceling pull, it decreases the flow by $\min \{-e(j), \bar{r}+e(i)\} \geq \bar{r} / 2$.

(2) A pull on arc $(i, j)$ increases only the absolute value of the deficit of node $i$. The new deficit of node $i$ is $e^{\prime}(i)=e(i)-\min \{-e(j), r(i, j), \bar{r}+e(i)\} \geq e(i)-(\bar{r}+e(i))=-\bar{r}$. Thus, $e^{\prime}(i) \geq-\bar{r}$ and $e_{\max } \leq \bar{r}$.

Theorem 3. For each node $i \in N, d(i)<2 n$. 
This theorem can be proved in a manner similar to the proof of the corresponding theorem from the complexity analysis of the generic preflow algorithm (for details see Ciurea and Ciupală 2001).

Theorem 4. During each scaling phase, the algorithm performs $O\left(n^{2}\right)$ noncanceling pulls.

Proof. We consider the potential function $F=-\sum_{i \in N} e(i) d(i) / \bar{r}$. The initial value of $F$ at the beginning of the $\bar{r}$-scaling phase is bounded by $2 n^{2}$ because $e(i) \geq-\bar{r}$ and $d(i) \leq 2 n$ for all $i \in N$ (from theorem 3). After the algorithm has selected node $j$, one of the following two cases must apply:

Case 1: The algorithm is unable to find an admissible arc along which it can pull flow. In this case the distance label of node $j$ increases by $q \geq 1$ units. This increases $F$ by at most $q$ units because $e(i) \geq-\bar{r}$. Since for each $i$ the total increase in $d(i)$ throughout the running of the algorithm is bounded by $2 n$, the total increase in $F$ due to relabelings of nodes is bounded by $2 n^{2}$.

Case 2: The algorithm is able to find an admissible arc along which it can pull flow, so it performs either a cancelling or a noncanceling pull. In either case, $F$ decreases. After a noncanceling pull on arc $(i, j)$, the flow from node $i$ to node $j$ decreases by at least $\bar{r} / 2$ units and $F$ decreases by at least $1 / 2$ units because $d(j)=d(i)+1$. As the initial value of $F$ at the beginning of the scaling phase plus the increase in $F$ sum to at most $4 n^{2}$, this case cannot occur more than $8 n^{2}$ times. Thus, the algorithm performs $O\left(n^{2}\right)$ noncanceling pulls per scaling phase.

Theorem 5. The deficit scaling algorithm runs in $O\left(n m+n^{2} \log \bar{c}\right)$ time.

Proof. Since the algorithm performs $O(\log \bar{c})$ scaling phase, from theorem 4 it follows that the algorithm performs $O\left(n^{2} \log \bar{c}\right)$ noncanceling pulls in total. The other operations (cancelling pulls, relabel operations and finding admissible arcs) require $O(\mathrm{~nm})$ time (this can be proved in a similar way as Ciurea and Ciupală (2001) proved the complexity of the generic preflow algorithm). Consequently, the deficit scaling algorithm runs in $O\left(n m+n^{2} \log \bar{c}\right)$ time.

\section{Conclusions and remarks}

In this paper, using the scaling technique, we developed a new implementation of the generic preflow algorithm for the minimum flow problem. Our algorithm is easy to understand and faster than the generic preflow algorithm and runs in $O\left(n m+n^{2} \log \bar{c}\right)$ time. Moreover, the deficit scaling algorithm can be easily parallelized in order to obtain an even quicker solution for the minimum flow problem.

Further research ideas: The scaling technique might be used to obtain more efficient algorithms for other classes of network flow algorithms, for example minimum cost flow algorithms, dynamic flow algorithms etc.

\section{References}

Ahuja R, Magnanti T, Orlin J 1993 Network flows. Theory, algorithms and applications (Englewood Cliffs, NJ: Prentice Hall)

Bang-Jensen J, Gutin G 2001 Digraphs: Theory, algorithms and applications (London: SpringerVerlag) 
Ciupală L, Ciurea E 2001 An approach to the minimum flow problem. Fifth Int. Symp. of Economic Informatics, pp 786-790

Ciupală L, Ciurea E 2003 An algorithm for the minimum flow problem. Sixth Int. Conf. of Economic Informatics, pp 565-569

Ciurea E, Ciupală L 2001 Algorithms for minimum flows. Comput. Sci. J. Moldova 9: 275-290

Ciurea E, Ciupală L 2004 Sequential and parallel algorithms for minimum flows. J. Appl. Math. Comput. 15: 53-78 